\title{
Laser In Endodontics-A Review Article
}

\author{
Dr.Pradnya V. Bansode ${ }^{1}$,Dr.Seema D.Pathak ${ }^{2}$,Dr.Madhuri Wavdhane ${ }^{3}$, \\ Dr.Shirish Khedgikar ${ }^{4}$,Dr.Shraddha Gite ${ }^{5}$ \\ I'Professor And Head Of Department, Department Of Conservative Dentistry And Endodontics, Gdc \& \\ Hospital, Aurangabad/ Muhs,India) \\ ${ }_{2}^{2}$ (Associate Professor, Department Of Conservative Dentistry And Endodontics, Gdc \& Hospital, Aurangabad/ \\ Muhs,India) \\ ${ }^{3}$ (Associate Professor, Department Of Conservative Dentistry And Endodontics, Gdc \& Hospital, Aurangabad/ \\ Muhs,India) \\ ${ }^{4}$ Lecturer, Department Of Conservative Dentistry And Endodontics, Gdc \& Hospital, Aurangabad/ Muhs, India) \\ ${ }^{5}\left(3^{\text {rd }}\right.$ Year Postgraduate Student,Department Dentistry And Endodontics, Gdc \& Hospital, Aurangabad/ \\ Muhs,India)
}

\begin{abstract}
Lasers were introduced into the field of clinical dentistry with the hope of overcoming some of the drawbacks posed by the conventional methods of dental procedures. Since its first experiment for dental application in the 1960s, the use of laser has increased rapidly in the last couple of decades. At present, wide varieties of procedures are carried out using laser.The purpose of this paper is to summarize laser applications in endodontics, including their use in pulp diagnosis, dentinal hypersensitivity, pulp capping and pulpotomy, sterilization of root canals, root canal shaping and obturation and Apicoectomy.The essential question is whether a laser can provide equal or improved treatment over conventional care. Secondary issues include treatment duration and cost/benefit ratio. This article reviews the role of lasers in endodontics and conservative dentistry since the early 1970s, summarizes many research reports from the last decade, and surmises what the future may hold for lasers in endodontics. With the potential availability of many new laser wavelengths and modes, much interest is developing in this promising field.
\end{abstract}

Keywords: Dentine; laser diagnosis; laser therapy use; root canal treatment

\section{Introduction}

The word "LASER" is widely used in dentistry. It stands for Light amplification by stimulated emission of radiation. With the rapid development of laser technology, newlasers with a wide range of characteristics are now available and being used in various fields of dentistry. Studies continue to be conducted in order to make maximum use of properties of the existing lasers in the field of endodontics. History Of Lasers In 1917, Einstein laid the foundation for the laser when he introduced the concept of stimulated emission; where a photon interacts with an excited molecule or atom and causes the emission of a second photon having the same frequency, phase, polarization and direction.

The Maser

A predecessor of the laser, called the MASER,for "Microwave Amplification by Stimulated Emission of Radiation", was independently developed in 1954 at Columbia University by Charles Townes and Jim Gordon and in Russia by Nicolay Basov and Alexandra Prokhorov.

\subsection{History Of Lasers In Dentistry}

The most recent development in endodontic treatment is the use of lasers. Since the development of the ruby laser by Maiman in 1960. Dental researchers began investigating lasers potential and Stern and Sognnaes reported in 1965 that a ruby laser could vaporize enamel.[1] The first laser use in endodontics was reported by Weichman\& Johnson (1971) who attempted to seal the apical foramen invitro by means of a high powerinfrared (CO2) laser. Subsequently, attempts were made to seal the apical foramen using the Nd:YAG laser.Practitioners and researchers began to find clinical oral soft tissue uses of medical CO2and Nd: YAG lasers until in 1990 when the first pulsed Nd: YAG laser designed specifically for the dental market was released. The year 1997 saw the FDA clearance of the first true dental hard tissue Er: YAG laser and the Er, Cr: YSGG a year later. Semiconductor based diode lasers emerged in the late 1990s as well

\subsection{Mechanism Of Action Of Lasers}

The word laser is an acronym standing for "Light Amplification of Radiation". A laser beam is created from a substance known as an active medium, which when stimulated by light or electricity produces photons of 
a specific wavelength. Lasers are characteristically monochromatic, unidirectional, coherent, and emitted from a stimulated active medium. Monochromacity means that a laser beam is made of a single wavelength of light, and all dental lasers are found in the visible or infrared portion of the electromagnetic spectrum. Once a laser beam is produced it will travel in one direction (unidirectional), though the divergence of the beam varies by type of laser and the associated transmission hardware. Coherence is the property that not only is a laser a single wavelength but all the peaks and valleys of each wave travel in unison.

The active media in dentistry can be solid state, gas, or semiconductor. Solid state lasers are a crystal matrix host doped with the light emitting, excitable atoms; such as erbium laced yttrium, aluminium, and garnet (Er: YAG). CO2is a popular laser where the active medium is sealed in an air tight chamber. Diode lasers have a semiconductor that when stimulated with electricity, laser light is emitted [2] Stimulated emission is a phenomenon that occurs within the active medium. For example, in solid state Er: YAG lasers the erbium is stimulated by light from a flash lamp with a process known as optical pumping. As an erbium atom absorbs a photon, its electrons are elevated to higher energy level. When the electrons return to a lower energy state, two identical photons are emitted and these photons can further stimulate more atoms in a chain reaction, resulting in amplification of the light produced. Mirrors surrounding the active medium called a resonator further increase this light energy. One of the mirrors called the output coupler is less than one hundred percent reflective. Light leaks from the output coupler and these are the photons that form the laser beam. Once the beam is created it is carried to the target tissue by various types of beam transfer hardware. Mirrors in articulated arms and optical fibers are common examples of this hardware[3]

Different lasers considered for endodontic applications are the near infrared laser-diode (810, 940, 980 and 1,064 nm) and Nd:YAG(1,064 nm) — and the medium infrared lasers-Erbium,Chromium: YSGG (Er,Cr:YSGG; 2,780 nm) and Erbium:YAG (2,940 nm).

Near infrared lasers such as Nd:YAG (from $803 \mathrm{~nm}$ to $1,340 \mathrm{~nm}$ ) were the first to be used for root optical fibre. The near infrared lasers are not absorbed by hard dentinal tissues and have no ablative effect on dentinal surfaces. The thermal effect of the radiation penetrates up to $1 \mathrm{~mm}$ into the dentinal walls, allowing for a decontaminating effect on deeper dentine layers.

The medium infrared lasers, such as the Erbium $(2,780 \mathrm{~nm}$ and 2,940 nm) laser family, with flexible,fine tips have also been used. The medium infrared lasers are well absorbed by the water content of the dentinal walls and consequently have a superficial ablative and decontaminating effect on the root canal surface.

The far infrared laser $\mathbf{C O 2}(10,600 \mathrm{~nm})$ was the first to be used in endodontics for decontamination and apical dentine melting in retrograde surgery. It is no longer used in this field with the exception of vitalpulp therapy (pulpotomy and pulp coagulation).

\section{Applications Of Lasers In Endodontics}

1. Lasers as a Diagnostic Tool for Endodontics

2. Analgesic Effects of Laser

3. Laser \& Dentin Hypersensitivity

4. Treatment of vital pulpal tissue-Pulpotomy andDirect pulp capping and pulp amputation

5. Root canal disinfection and irrigation-

- Access cavity preparation and root canal orifice enlargement.

- Root canal wall preparation.

- Sweeping of Root canal and irrigation.

- Sterilization or disinfection of infected canals.

- Obturation with gutta percha or resin.

- Removal of temporary cavity sealing materials, root canal sealing materials, and fractured instruments in root canals

6. Vertical root fracture diagnosis and treatment

7. Laser Assisted Obturation and removal of gutta percha obturation material.

8. Endodontic surgery-

- Flap preparation - incision of soft tissue to prepare a flap and expose the bone.

- Cutting bone to prepare a window access to the apex (apices) of the roots

- Apicoectomy - amputation of the root end

- Root end preparation for retro fill amalgam or composite

- Removal of pathological tissues (i.e., cysts, neoplasm or abscess) and hyperplastic tissues (i.e., granulation tissue) from around the apex. 


\section{Laser \& Dentin Hypersensitivity}

The use of lasers in endodontics has been studied since the early 1970s, and lasers have been morewidely used since the 1990s. A successful endodontic therapy is when there is complete and effectivecleaning of root canal.[4]Tradi-tional endodontic techniques use mechanical instru-ments, as well asultrasound and chemical irrigation to shape, clean and completely decontaminate the endodonticsystem. There is limitation of endodontic therapy such as lateral canals with various morphologies and dimensions. [5] The usefulness of the debridement, cleaning and refining of the intra-radicular space is limited, because of anatomical complexity and the difficulty of common irrigants to penetrate into the lateral canals and the apical ramifications. Hence, there is need for new materials, tech- niques and technologies that can improve the cleaning and decontamination of these anatomical areas.[6]

Different wavelengths have been shown to be effective in significantly reducing bacteria in infected canals and studies have confirmed these results in vitro. Further studies have demonstrated the efficiency of lasers in combination with commonly used irrigants, such as 17\% EDTA, 10\% citric acid and 5.25\% sodium hypochlorite.[7]The action of the chelating substances facilitates the penetration of laser light, which can penetrate into the dentinal walls up to $1 \mathrm{~mm}$ in depth and have a stronger decontaminating effect than chemical agents. Laser-activated irrigation, has been shown to be statistically more effective in removing debris and the smear layer in root canals compared with traditional techniques and ultrasound.[8]A recent study by DiVito et al. demonstrated that the use of the Erbium laser at sub-ablative energy density using a radial and stripped tip in combination with EDTA irrigation results in effective debris and smear layer removal without any thermal damage to the organic dentinal structure. In endodontics, lasers use the photo- thermal and photomechanical effects resulting from the interaction of different wavelengths and different parameters on the target tissues. These are dentine, the smear layer, debris, residual pulp and bacteria in all their various aggregate forms.

Laser Wavelength Consideration[9]-The primary use of lasers in endodontics is focused on eradicating micro-organisms in the root channel, especially in the lateral dentinal tubuli. This requires a wavelength that shows high transmission through hydroxyapatite and water. Absorption curves show that Nd:YAG lasers, and in particular pulsed Nd:YAG lasers, are first-choice for this application. Nd:YAG lasers show the best results in transmission and microorganism reduction measurements.

\section{Thermal Consideration}

Behrens and Gutknecht, 1993,[10]conducted in- vitro experiments on dentine slices with laser power settings that take into account even the most extreme situations, in order to determine that no thermal damage occurs in pulsed Nd: YAG laser or diode laser treatments. When measuring the root surface, a temperature of $38^{\circ} \mathrm{C}$ was obtained after a 45 -second treatment duration at $15 \mathrm{~Hz} / 1.5 \mathrm{~W}$. This value lies within the physiological area. It must be considered that in an in-vivo situation the dental tissue is more efficiently cooled by the blood flow that surrounds the root surface.

\section{Morphological Changes}

The smear layer is completely removed and the dental tubuli are, for the most part closed through inorganic melting if the Nd:YAG laser is applied with $15 \mathrm{~Hz} / 1.5 \mathrm{~W}$ settings.[11] Similar results can be expected if the $810 \mathrm{~nm}$ laser diode is used. If the Er:YAG laser is applied, the smear layer will be completely removed and the dental tubuli remain open.

\section{Disinfection Effect}

GUTKNECHT et al[12] achieved an average of $99.92 \%$ bacterial reduction in the root canal using the Nd:YAG laser. In 1994, Rooney et aLand Hardee et al described reductions of $99 \%$ when using a Nd: YAG laser in different experimental designs and bacterial combinations. Further studies examined the depth effect of the laser in the root canal dentine. In 1997, Klinke et al were able to prove a bactericidal effect of the Nd:YAG laser at a depth of $1,000 \mu \mathrm{m}$. In comparison, a rinsing solution, such as $\mathrm{NaOCl}$, only achieves effective bacterial reduction up to a depth of $100 \mu \mathrm{m}$.

Indications and contra-indications for laser supported endodontic treatments [13]

Laser-supported treatments should be favored when treating patients that show one or several of the following symptoms:

1. Teeth with a purulent pulpitis or pulp necrosis

2. Teeth, of which the crown and root pulp show gangrenous changes

3. Teeth with peri-apical lesions (peri-apical gap from $1 \mathrm{~mm}$, up to granulomas with a diameter of $5 \mathrm{~mm}$ and more

4. Teeth with a peri-apical abscess 
5. Teeth with lateral canals that lead to periodontal involvement

6. Absorption of the apex caused by inflammation or trauma

7. Teeth that have been treated for at least threemonths without success (with alternating rinsing and medicinal inlay

2.1 Laser as a Diagnostic tool in endodontics-Determination of pulp vitality-

Laser doppler flowmetry is a device used to determine pulp vitality based on red blood cell flux in the pulp tissue. It is particularly useful for the detection of pulp vitality in traumatized and/or immature teeth (Singh et al., 2013)

\section{Mechanism of Action-}

Light beams from the optical fibre enters the tissue, is absorbed to some extent by the blood cells and another fibre tip collects the scattered light and provides information about pulp vitality

Its advantages are non-invasive, painless diagnosis of the tooth , reproducible, gold standard for pulpal blood flow determination .

Limitations are- takes longer than other vitality determination techniques, requires a special device. Therefore, it is not conducted as a routine procedure in clinical practice

\subsection{Lasers in Pulp Capping[14]- for Indirect Pulpcapping-}

Pulsed Nd:YAG laser is used and black ink applied on the tooth surface. Air spray cooling is needed to prevent pulp damage resulting from the laser energy. $\mathrm{CO}_{2}$ laser can also be used. In some cases, it is recommended that this laser be used with $38 \%$ silver ammonium solution. These treatments should be performed under local anesthesia.

\subsection{Direct pulp capping by laser[14]}

$\mathrm{CO}_{2}$ laser irradiation is performed at 1 or $2 \mathrm{~W}$ after irrigating with $8 \%$ sodium hypoclorite and $3 \%$ hydrogen peroxide for more than 5 minutes. Calcium hydroxide paste must be used to dress the exposed pulp after laser treatment, after which the cavity should be tightly sealed with cement such as polycarboxylate cement. Pulsed Nd: YAG, argon, semiconductor diode, and Er:YAG can also be used.

Laser Ablation and Accessory Treatment for Vital Pulp Amputation[14]-The lasers used are CO2, pulsed Nd:YAG, He-Ne and low power semiconductor diode lasers and middle power semiconductor diode lasers. $\mathrm{CO} 2$ laser usage is time consuming and pulp tissue may be damaged due to several exposures. Pulsed Nd: YAG causes damage to the pulp tissue and thereby showed a low success rates so it should be used only for pulp hemostasis, sedation, antinflammatory effects, and stimulation of remaining pulpal cells.

\subsection{Laser \& Dentin Hypersensitivity}

The mechanism of laser for treatment of dentin hypersensitivity is not well explained, However Pashley suggests that it may occur through coagulation and protein precipitation of plasma in dentinal fluid or by alteration of nerve fiber activity. 8 Laser therapy has been recommended by Kimura etal. to treat dentin hypersensitivity with effectiveness between $5.2 \%$ and $100 \%$, depending on the type of laser and parameters used. According to authors, lasers are more effective than other treatments,although effect diminishes in severe dentin hypersensitivity. Mckarthy et al indicates that thereduction in dentin hypersensitivity could be the result of alteration of root dentin surface, physically occluding the dentinal tubules.

\subsection{Laser in Analgesia}

The pulsed â Nd:YAG laser is widely used as a analgesia in endodontics. Its wavelengths interfere with the sodium pump mechanism, change cell membrane permeability, alter temporarily the endings of sensory neurons, and block depolarization of $\mathrm{C}$ and $\mathrm{A}$ fibers of the nerves.

\subsection{Root canal disinfection and irrigation-The various uses of laser in root canal treatments are as follow}

1. Access cavity preparation and root canal orifice enlargement.

2. Root canal wall preparation.

3. Sweeping of Root canal and irrigation.

4. Removal of pulp remnants and debris at the apical foramen.

5. Sterilization or disinfection of infected canals.

6. Obturation with gutta percha or resin.

7. Removal of temporary cavity sealing materials, root canal sealing materials, and fractured instruments in root canals. 
- Access cavity preparation- Er,Cr:YSGG (2780nm) and Er:YAG (2940nm) can be used for access cavity preparation, root canalshaping and cleaning.

- Root canal wall preparation-Lasers such as Er:YSGG (2780nm), Er:YAG(2940nm) and Nd:YAG(1064nm) are use $3 \mathrm{~d}$ for root canal wall preperation ${ }^{15}$. When the laser fiber is unable to be inserted into thecanals, reamers and files are to be used, followed by lasers. Smear layer is completely removed and dentinal tubuli are for the most part closed if pulsed Nd:YAG laser is applied at $15 \mathrm{~Hz} / 1.5 \mathrm{~W}$ settings.

- Sweeping of Root canal and irrigation are done in Straight, slightly curved and wide canals with lasers.Along with lasers, 5.25\% Sodium hypochlorite or $14 \%$ EDTA must be used along laser irradiation.Nd:YAG are widely used for removal of pulp remnants and debris at the apical foramen. Removal of pulp remnants, control of hemorrhage, and stimulation of cells surrounding the root apex as well as debridement on the surface.

- Sterilization or disinfection of infected canals are done with Pulsed Nd:YAG, argon, semiconductordiode, $\mathrm{CO}_{2}$, Er:YAG lasers. Because of laser energy and wavelength characteristic, they are useful in killing microorganism.

- In endodontics, lasers use the photo-thermal and photomechanical effects resulting from the interaction of different wavelengths and different parameters on the target tissues.

With vertical condensation method, obturation of canals can be done with Lasers. Anic and Matsumoto attempted to investigate whether it is possible to perform the root canal filling using sectioned gutta-percha segments and a pulsed Nd:YAG laser. With the lasers, Removal of temporary cavity sealing materials, root canal sealing materials, and fractured instruments in root canals became possible.15 In fine and strongly curved canals, however, there were many cases in which laser tips perforated the canal wall.

\subsection{Laser in Apicoectomy, Retrograde and Endodontic Apical Cavity Preparation, and}

Periapical Curettage -The advantages of laser application in endodontic surgery are identical to those that have been reported for other oral surgical procedures. Soft tissue lasers like the Nd:YAG; Diode or CO2 can be used for providing clean incisions for gaining direct access to the periradicular region. In addition, the use of lasers to replace aerosol-producing handpieces in periapical surgery can reduce the risk of contamination of the surgical environment by blood borne pathogens. The Er:YAG or the Er,Cr:YSGG lasers can be used for cutting into bone as well as sectioning the apical 3rd for apicoectomy \& have also been researched for retro-preparation. The unique properties of laser light as they pertain to endodontic surgery are listed as follows: precision; coagulation; decreased postoperative pain, edema and reduced scarring; sterilization; selective absorption.

\subsection{Vertical root fracture diagnosis and treatment-}

There are very few studies examining the use of lasers in diagnosing vertical root fracture cases. Although Kimura et al. (2009) used diagnodent for vertical root fracture detection in vivo, this technique appears to be impractical for clinical use. Vertical fractures can be treated using a surgical approach on the fracture side, which involves cleaning of the fracture line and filling with composite resin or bioactive materials. Thereafter, lasers can be used to accelerate soft tissue repair, and this has been performed previously using low power laser therapy (Nogueira et al., 2012)

\subsection{Laser Assisted Obturation:}

Due to their thermal effects clinicians have also tried using lasers like the Nd:YAG for thermosoftening gutta-percha; however there isn't much of an advantage seen over the thermoplasticised gutta-percha obturations systems commercially available today. Nd:YAG laser has also been used for sectioning excess gutta percha left after lateralcompaction techniques.

Indications.

\section{III.Indication And Contraindication Of Lasers In Endodontics}

a. Teeth with lateral canal leading to periodontal involvment.

b. Teeth with pulp necrosis and purulent pulpitis.

c. Teeth with gangarenous changes.

d. Teeth with periapical lesions upto $5 \mathrm{~mm}$ or more.

e. Teeth that has been treated atleast 3 months with no success.

\section{Contraindications}

a.In advanced periodontitis cases.

b. A deep crown and root fracture. 
c. Obliterated root canals in endodontic treated teeth.

\section{Conclusion}

It is concluded that with the advent of Lasers in dentistry, the complex procedures have become easier and time saving. Thus the patient care has improved.

\section{Acknowledgements}

It's my immense pleasure to express my deep sense of gratitude and sincere thanks to my Dean Dr. S. P. Dange, Government Dental College \& Hospital Aurangabad

\section{References}

[1]. Stern RH, Sognnaes RF. Laser Effect on Dental Hard Tissues. A Preliminary Report.J South Calif Dent Assoc 1965;33:17-9

[2]. Adrian JC, Bernier JL, Sprague WG. Laser and the dental pulp. J Am Dent Assoc 1971 ;83(1):113-7.6.

[3]. Lin S, Liu Q, Peng Q, Lin Ml. The ablation threshold of Er:YAG laser and Er,Cr:YSGG laser in dental dentin. Scientific Research and Essays 2010;5(16):2128-35.7.Jesse J, Desai S, Oshita P

[4]. Koukichi Matsumoto: Lasers in Endodontics:DCNA. 2000; Vol 44(4): 889-906.

[5]. Giovanni Olivi, Rolando Crippa, Giuseppe Iaria,Vasilios Kaitsas, Enrico DiVito \& Stefano

[6]. Benedicenti. Lasers in endodontics ( Part I). Roots; 2011:1-4.

[7]. Karlovic Z, Pezelj-Ribaric S, Miletic I, Jukic S,Grgurevic J, Anic I. Erbium:YAG laser versus ultrasonic in preparation of root-end cavities. JEndod 2005;31:821-3.

[8]. Anic I, Matsumoto K: Comparison of the sealing ability of laser softened, laterally condensed and low temperature thermoplasticized gutta percha.J Endod .1995;21:464-469.

[9]. K Gorkhay et al: Effects of oral soft tissue produced by a diode laser in vitro. Lasers in

[10]. Surgery and medicine 1999; 25:401-406.

[11]. Proceedings of the 1st International Workshop of Evidence Based Dentistry onLasers in Dentistry, Quintessence Publishing, 2007, ISBN 978-1- 85097-167-2. 18.

[12]. Gutknecht N, Franzen R, Lampert F. FiniteElement Study on Thermal Effects in Root Canals During Laser Treatment with a Surface-absorbed Laser. Lasers Med Sci2002;17:137-44.

[13]. Blöschl G, Kirnbauer R, Gutknecht D.Distributed snowmelt simulations in an Alpine catchment. 1. Model evaluation on the basis of snow cover patterns. WaterResources Research 1991;27(12):3171-9.

[14]. Gutknecht N, Moritz A, Conrads G, SievertT, Lampert F. Bactericidal effect of the Nd:YAG laser in in vitro root canals. J ClinLaser Med Surg 1996;14:77-80.

[15]. Gutknecht N. Lasers in Endodontics. Journal of the Laser and Health Academy 2008;4:1-8.

[16]. Mathew S, Thangaraj DN. Lasers In Endodontics. JIADS 2010;1(1):31-7.

[17]. Kathari A, Ujariya M. Lasers in endodontics- A review. J Res Adv Dent 2014; 3:1:209-211.

\section{Indentations and EQUATIONS}

The first paragraph under each heading or subheading should be flush left, and subsequent paragraphs should have a five-space indentation. A colon is inserted before an equation is presented, but there is no punctuation following the equation. All equations are numbered and referred to in the text solely by a number enclosed in a round bracket (i.e., (3) reads as "equation 3"). Ensure that any miscellaneous numbering system you use in your paper cannot be confused with a reference [4] or an equation (3) designation. (10)

\section{Figures and Tables}

To ensure a high-quality product, diagrams and lettering MUST be either computer-drafted or drawn using India ink.

Figure captions appear below the figure, are flush left, and are in lower case letters. When referring to a figure in the body of the text, the abbreviation "Fig." is used. Figures should be numbered in the order they appear in the text.

Table captions appear centered above the table in upper and lower case letters. When referring to a table in the text, no abbreviation is used and "Table" is capitalized. (10)

\section{Conclusion}

A conclusion section must be included and should indicate clearly the advantages, limitations, and possible applications of the paper. Although a conclusion may review the main points of the paper, do not replicate the abstract as the conclusion. A conclusion might elaborate on the importance of the work or suggest applications and extentions. (10)

\section{Acknowledgements}

An acknowledgement section may be presented after the conclusion, if desired.( 8) 


\section{This heading is not assigned a number.}

\section{References}

A reference list MUST be included using the following information as a guide. Only cited text references are included. Each reference is referred to in the text by a number enclosed in a square bracket (i.e., [3]). References must be numbered and ordered according to where they are first mentioned in the paper, NOT alphabetically.

\section{Examples follow:}

Journal Papers:

[1]. M Ozaki, Y. Adachi, Y. Iwahori, and N. Ishii, Application of fuzzy theory to writer recognition of Chinese characters, International Journal of Modelling and Simulation, 18(2), 1998, 112-116. (8)

[2]. Note that the journal title, volume number and issue number are set in italics.

Books:

[3]. R.E. Moore, Interval analysis (Englewood Cliffs, NJ: Prentice-Hall, 1966). (8)

[4]. Note that the title of the book is in lower case letters and italicized. There is no comma following the title. Place of publication and publisher are given.

Chapters in Books:

[5]. P.O. Bishop, Neurophysiology of binocular vision, in J.Houseman (Ed.), Handbook of physiology, 4 (New York: Springer-Verlag, 1970) 342-366. (8)

[6]. Note that the place of publication, publisher, and year of publication are enclosed in brackets. Editor of book is listed before book title.

Theses:

[7]. D.S. Chan, Theory and implementation of multidimensional discrete systems for signal processing, doctoral diss., Massachusetts Institute of Technology, Cambridge, MA, 1978. (8)

[8]. Note that thesis title is set in italics and the university that granted the degree is listed along with location information

Proceedings Papers:

[9]. W.J. Book, Modelling design and control of flexible manipulator arms: A tutorial review, Proc. 29th IEEE Conf. on Decision and Control, San Francisco, CA, 1990, 500-506 (8) 